\title{
Optimization of Maxillary Obturator Thickness Using a Double-Processing Technique
}

\author{
Won-suck Oh, DDS, MS ${ }^{1} \&$ Eleni D. Roumanas, DDS ${ }^{2}$ \\ ${ }^{1}$ Associate Clinical Professor, Division of Prosthodontics, Department of Biologic \& Materials Sciences, University of Michigan School of Dentistry, \\ Ann Arbor, Ml \\ ${ }^{2}$ Professor, Division of Advanced Prosthodontics, Biomaterials and Hospital Dentistry, and Director, Graduate Prosthodontics, The Jane and Jerry \\ Weintraub Center for Reconstructive Biotechnology, UCLA School of Dentistry, Los Angeles, CA
}

\author{
Keywords \\ Prosthetic rehabilitation; obturator prosthesis; \\ double-processing technique.

\section{Correspondence} \\ Won-Suck Oh, University of Michigan School \\ of Dentistry, 1011 N. University, Ann Arbor, \\ MI 48109-1078. E-mail: \\ ohws@umich.edu
}

Accepted June 20, 2006.

doi: 10.1111/j.1532-849X.2007.00249.x

\begin{abstract}
Preservation of remaining structures is a primary goal of prosthetic rehabilitation. Continuously applied stresses on the remaining tissues from a large, heavy obturator jeopardize the health of the tissues, compromise the function of the prosthesis, and affect patient comfort. Various techniques have been described for hollowing the bulb of an obturator after processing to reduce its weight; however, access to the inner aspects of the bulb is limited, preventing adequate control of thickness of the walls. This article describes a double-processing technique for an obturator to optimize the weight and thickness of the bulb.
\end{abstract}

Ablative surgical therapy is frequently adopted for the control of malignancies and other abnormal growths within the maxillary sinuses. This creates an anatomic defect that allows the oral cavity, maxillary sinus, and nasal cavity to become one compartment. ${ }^{1}$ Prosthetic rehabilitation with an obturator prosthesis is a predictable intervention to recreate an anatomic barrier between the cavities and to restore functional capabilities of speech, oral food intake, and deglutition. $^{2-5}$

The degree of extension into the defect varies depending upon the configuration of the defect, character of its lining tissue, and functional requirements for retention, support, and stabilization of the prosthesis. ${ }^{6}$ In large defects lacking palatal support, the obturator is aggressively extended vertically to engage the surgical defect and horizontally to the lateral aspect of the orbital floor, at the expense of its size and weight. Remaining structures are subjected to continuous stresses from such large, heavy obturators, jeopardizing the health of the tissues, and compromising patient function and comfort. ${ }^{7,8}$ To reduce the weight of the prosthesis, the bulb portion of the obturator is generally hollowed after it has been processed into acrylic resin. Weight reduction is especially important when the obturator prosthesis is suspended without bony or posterior tooth support on the defect side, as is the case with most maxillary resection prostheses. ${ }^{9}$ A hollow maxillary obturator may reduce the weight of the prosthesis by up to $33 \%$, depending upon the size of the maxillary defect. ${ }^{10}$

The obturator is conventionally hollowed through a small opening on the superior or palatal aspect of the bulb, as described in various techniques. ${ }^{11-13}$ This approach restricts access to the inner aspect of the bulb and limits the degree of thinning that can be achieved. This article describes a doubleprocessing technique for a maxillary obturator which controls and optimizes the weight and thickness of the bulb.

\section{Technique}

1. Following physiologic adjustment of the metal framework in the mouth, a custom tray is border molded, and a functional impression is made to capture the complex contours of the surgical defect. ${ }^{14}$ This altered cast procedure is employed to relate the soft tissue contours to the remaining abutment teeth (Fig 1).

2. A layer of baseplate wax (1.5-mm thick) is adapted onto the walls of the surgical defect and extended to the finish line of the metal framework. Care should be taken to block out all undercuts with wax and to prevent fracture of the stone mold during flask opening and trial packing procedures. The master cast is flasked, packed in the customary manner, and processed using heat-activated 

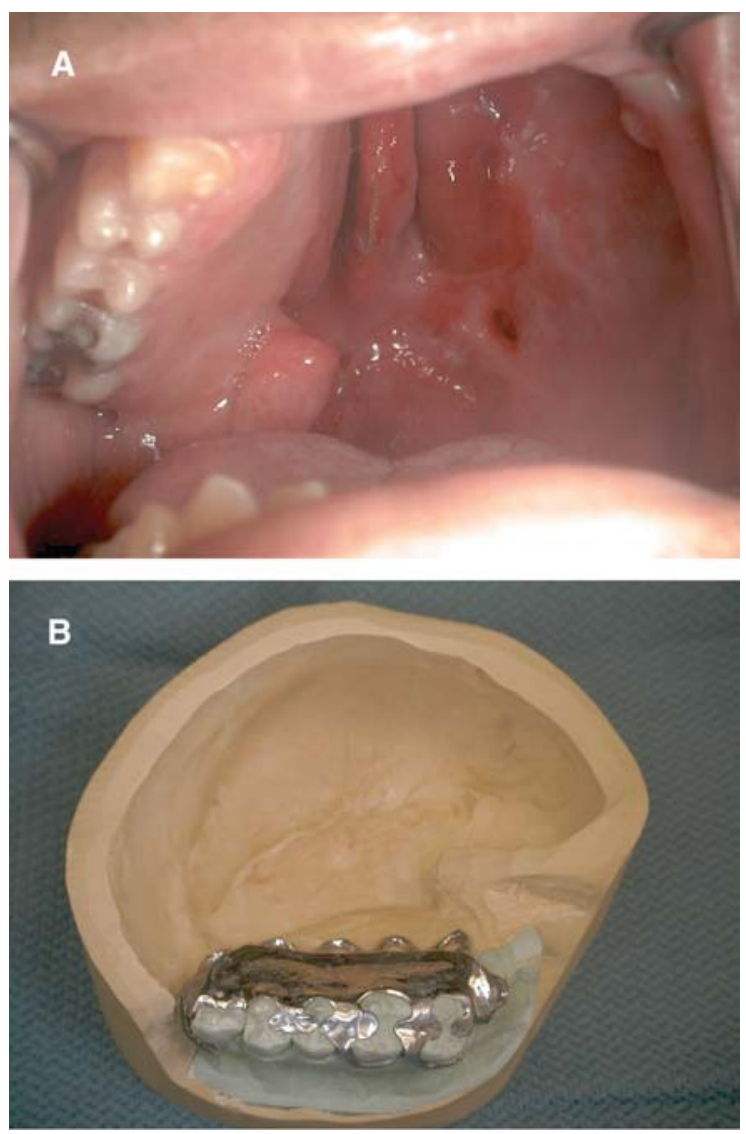

Figure 1 (A) Postoperative intraoral view of the surgical defect, residual palatal shelf, and remaining dentition. (B) Altered master cast with metal framework.

acrylic resin (Lucitone 199, Dentsply Intl, Alsip, IL) in a curing tank set at $165^{\circ} \mathrm{F}$ for 9 hours (Fig 2).

3. The record base is divested, and an opening is made in the top surface. Acrylic denture burs are used to thin the internal aspects of the record base through both the superior and palatal openings (Fig 3A). Care should be taken not to overthin the bulb, as perforation may occur during the clinical try-in and adjustment of pressure areas. Following adjustments, palatal contours are formed with a layer of baseplate wax, and a wax occlusion rim is added (Fig 3B). Wax contours are adjusted for proper articulation and occlusal vertical dimension. Registration of the maxillomandibular jaw relationship is made using a centric relation record.

4. Laboratory silicone putty impression material (LabPutty, Coltène/Whaledent, Inc., Cuyahoga Falls, OH) is adapted to create a remount index and to stabilize and retain the processed record base to the original cast. Vertical and horizontal jaw relations are registered, and the casts are mounted on an articulator (Fig 4). Palatal contour is refined during the final try-in of the wax obturator, based on tongue and palate interaction.
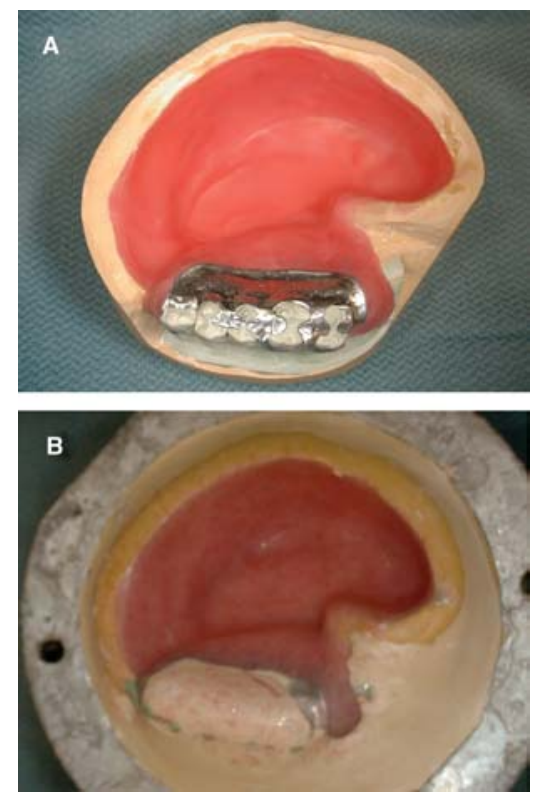

Figure 2 (A) Adaptation of a layer of baseplate wax to the surgical defect and residual palatal shelf area. (B) Heat-processed record base in the investment.
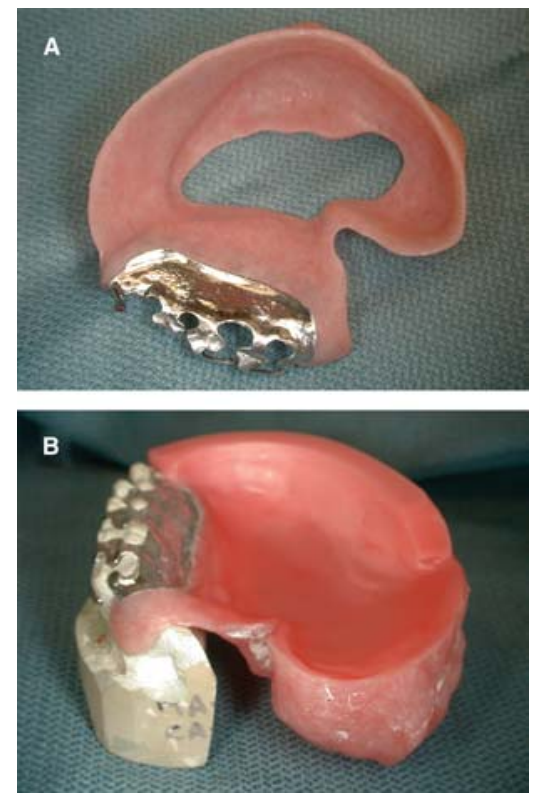

Figure 3 (A) Trimmed, heat-processed record base with openings in the palatal and superior aspects. (B) Closure of palatal opening with a layer of baseplate wax and build-up of wax occlusion rim.

5. Once tooth position and palatal contours have been optimized, the obturator is festooned, and the internal aspect of the bulb is lined with dental stone and packed with silicone putty (Lab-Putty). The obturator is then flasked, wax is boiled out, and the obturator is subjected to a second processing procedure using heat-activated acrylic resin (Lucitone 199) at $138^{\circ} \mathrm{F}$ for 12 hours 


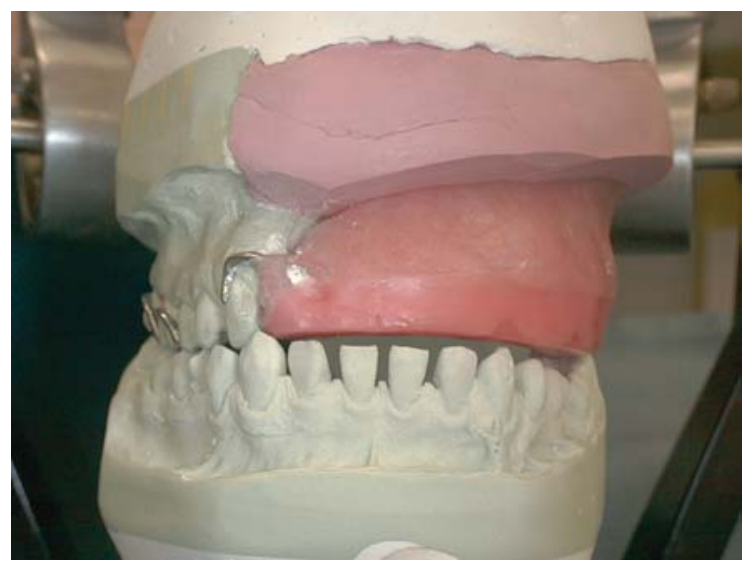

Figure 4 Remount index fabricated with silicone putty impression material for mounting of the master cast and processed record base on an articulator.

(Fig 5A, B). Note the thin and neatly finished definitive obturator (Fig 5C,D).

\section{Discussion}

In dentate patients, primary retention, support, and stability of an obturator depends on the number and distribution of remaining teeth. ${ }^{2-5}$ Engagement of soft tissue undercuts, including the scar band at the skin graft-mucosal junction, may also play a significant role, particularly in edentulous patients. ${ }^{6}$ Wide surgical resections for the control of malignancies frequently result in a small number of remaining, unilaterally clustered teeth. ${ }^{1}$ These remaining teeth serve as abutments for the obturator and are subjected to constant, nonaxial, cantilever forces. ${ }^{7,8}$ The skin graft-mucosal junction scar band will also stretch out over time and become ineffective in helping to retain the obturator.

The weight of an obturator can be significantly reduced by hollowing out the bulb. ${ }^{10}$ The importance of this procedure can be neglected and compromised due to restricted access and the difficulty of hollowing out the bulb. ${ }^{11-13}$ A processed record base with holes on both the superior and palatal aspects allows convenient access for further reducing the obturator bulb thickness. Using this technique, the thickness of obturator walls and palate are reduced to less than $1 \mathrm{~mm}$, resulting in a significant decrease in weight. Vertical and horizontal extension of the lateral walls of the obturator can be maximized without additional increase in weight. The degree of obturator movement is minimized by improving obturator-tissue contact superiorlaterally. ${ }^{6}$ Abutment teeth and soft tissue undercuts will be subject to less stress to meet the primary goal of prosthodontic rehabilitation, "preservation of the remaining structure."15

Structural durability is mandatory for longevity of the prosthesis. In these patients, chewing function is confined to the nonsurgical side due to the lack of support on the surgical defect side. The bulb portion of the obturator is designed so that the 3D configuration of congruous vertical and horizontal walls offset crack development and fracture propagation. Tensile stress accumulation developing along the midline of conventional complete dentures ${ }^{16}$ resulting in fatigue fracture is not a problem in the case of maxillary obturators.

A stable record base is critical for recording an accurate maxillomandibular relationship and in evaluation of the esthetics and phonetics of the wax trial prosthesis. ${ }^{17,18}$ The fit and stability of the record base for an obturator, however, is often compromised because of the size of the surgical defect and is further compounded by the need to block out undercuts and by under-extended borders. Conventional record bases can rotate into the defects when attempting the centric relation
Figure 5 (A) Palatal aspect of wax obturator in investment. (B) Record base and stone core after boil-out of wax. (C) Lateral view of finished obturator. (D) Frontal view of finished obturator.
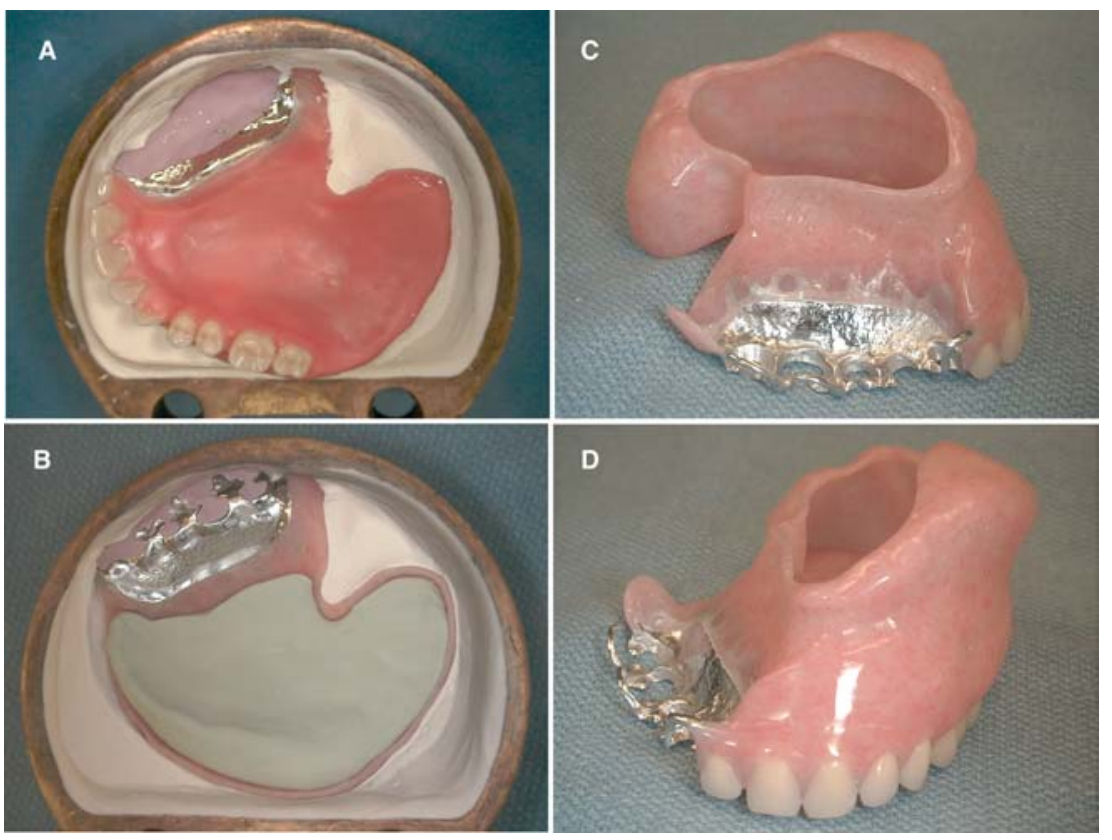
record, resulting in an inaccurate record. A processed record base provides maximum support, retention, and stability for a maxillofacial prosthetic patient ${ }^{18}$ and additional steps of relining the record base with silicone resilient materials can be avoided. ${ }^{19}$

Depending upon the nature of the defect, movement of the obturator varies and creates soreness and discomfort for the patient. These pressure sores are adjusted at the postinsertion and subsequent follow-up appointments. To avoid the risk of perforation of the bulb, final contouring is accomplished after the patient is completely comfortable with the new obturator prosthesis. Patient benefits from the reduced weight of the obturator offset the costs and additionally incurred laboratory procedures for two-stage processing. Employing a lower temperature and a longer processing cycle than that used to process the record base minimizes dimensional change of the record base. ${ }^{20}$ This technique can also be applied to completely edentulous patients.

\section{References}

1. Spiro RH, Strong EW, Shah JP: Maxillectomy and its classification. Head Neck 1997;19:309-314

2. Beumer J, Curtis TA, Marunick MT: Maxillofacial Rehabilitation Prosthodontic and Surgical Considerations, 2nd edition. St. Louis, MO, Ishiyaku Euroamerica Inc, 1996, pp. 225-247

3. Umino S, Masuda G, Ono S, et al: Speech intelligibility following maxillectomy with and without a prosthesis: An analysis of 54 cases. J Oral Rehabil 1998;25: 153-158

4. Rieger J, Wolfaardt J, Seikaly H, et al: Speech outcomes in patients rehabilitated with maxillary obturator prostheses after maxillectomy: A prospective study. Int J Prosthodont 2002;15:139-144

5. Sullivan M, Gaebler C, Beukelman D, et al: Impact of palatal prosthodontic intervention on communication performance of patients' maxillectomy defects: A multilevel outcome study. Head Neck 2002;24:530-538

6. Brown KE: Peripheral consideration in improving obturator retention. J Prosthet Dent 1968;20:176-181

7. Schwartzman B, Caputo A, Beumer J: Occlusal force transfer by removable partial denture designs for a radical maxillectomy. $\mathrm{J}$ Prosthet Dent 1985;54:397-403

8. Schwartzman B, Caputo AA, Beumer J: Gravity-induced stresses by an obturator prosthesis. J Prosthet Dent 1990;64:466-468

9. Minsley GE, Nelson DR, Rothenberger SL: An alternative method for fabrication of a closed hollow obturator. J Prosthet Dent 1986;55:485-490

10. Wu YL, Schaaf NG: Comparison of weight reduction in different designs of solid and hollow obturator prostheses. J Prosthet Dent 1989;62:214-217

11. Chalian VA, Barnett MO: A new technique for constructing a one-piece hollow obturator after partial maxillectomy. J Prosthet Dent 1972;28:448-453

12. Knapp JG: A simplified approach to the fabrication of a maxillary hollow obturator prosthesis. J Prosthet Dent 1984;51:67-69

13. Jhanji A, Stevens ST: Fabrication of one-piece hollow obturators. J Prosthet Dent 1991;66:136-138

14. Oh W, Roumanas E: Alternate technique for fabrication of a custom impression tray for definitive obturator construction. J Prosthet Dent 2006;95:473-475

15. DeVan MM: The concept of neutrocentric occlusion as related to denture stability. J Am Dent Assoc 1954;48:165-169

16. Vlissidis D, Paipetis SA: The mechanical strength of maxillary complete dentures. J Biomed Mater Res 1984;18:413-425

17. Langer A: The validity of maxillomandibular records made with trial and processed acrylic resin bases. J Prosthet Dent 1981;45:253-258

18. Jacob RF, Yen TW: Processed record bases for the edentulous maxillofacial patient. J Prosthet Dent 1991;65:680-685

19. Murata H, Hamada T, Nagasiri R: Stabilizing record bases for edentulous obturator prostheses with silicone resilient relining material. J Prosthet Dent 1999;82:366-368.

20. Graser GN: Completed bases for removable dentures. J Prosthet Dent 1978;39:232-236 